\title{
Contrasting functions of the epithelial-stromal interaction 1 gene, in human oral and lung squamous cell cancers
}

\author{
MENGMENG FAN $^{1}$, MAKOTO ARAI ${ }^{1}$, AKINOBU TAWADA ${ }^{1}$, TETSUHIRO CHIBA ${ }^{2}$, REO FUKUSHIMA ${ }^{3}$, \\ KATSUHIRO UZAWA $^{3}$, MASASHI SHIIBA ${ }^{1}$, NAOYA KATO ${ }^{2}$, HIDEKI TANZAWA $^{3}$ and YUICHI TAKIGUCHI $^{1}$ \\ Departments of ${ }^{1}$ Medical Oncology, ${ }^{2}$ Gastroenterology and ${ }^{3}$ Oral Science, \\ Graduate School of Medicine, Chiba University, Chiba 260-8670, Japan
}

Received September 17, 2020; Accepted December 23, 2020

DOI: $10.3892 /$ or.2021.8216

\begin{abstract}
The epithelial-stromal interaction 1 gene (EPSTII) is known to play multiple roles in the malignant progression of breast cancer and also in some aspects of the immune responses to the tumor. However, the relevance of the gene in the onset/progression of oral squamous cell carcinoma (OSCC) and lung squamous cell carcinoma (LSCC) is not yet known. The present study was aimed at revealing the roles of EPSTI1 in conferring malignant characteristics to OSCC and LSCC, and the underlying mechanisms. Quantitative real-time polymerase chain reaction (PCR) and western blot analyses demonstrated significant upregulation of EPSTI1 in all four OSCC cell lines (HSC2, HSC3, HSC3-M3 and HSC4), and significant downregulation of EPST11 in all three LSCC cell lines (LK-2, EBC-1 and H226) used in the present study, as compared to the expression levels in the corresponding control cell lines. Both knockdown of EPST11 in OSCC and overexpression of the gene in LSCC suppressed cell proliferation, and induced cell-cycle arrest in the G1 phase, with upregulation of $p 21$ and downregulation of $C D K 2$ and cyclin D1. Furthermore, these alterations of EPST11 gene expression in the OSCC and LSCC cell lines suppressed the cell migration ability and reversed the EMT phenotype of the tumor cells. Collectively, while EPSTI1 appears to have oncogenic roles in OSCC, it appears to exert tumor-suppressive roles in LSCC. PCR array analyses revealed some genes whose expression levels were altered along with the modified EPSTII expression in both the OSCC and LSCC cell lines. These findings suggest that EPSTI1 may be a therapeutic target for both OSCC and LSCC.
\end{abstract}

Correspondence to: Professor Yuichi Takiguchi, Department of Medical Oncology, Graduate School of Medicine, Chiba University, 1-8-1 Inohana, Chuo-ku, Chiba 260-8670, Japan

E-mail: takiguchi@faculty.chiba-u.jp

Key words: EPSTI1, oral cancer, lung cancer, squamous cell carcinoma, epithelial-mesenchymal transition

\section{Introduction}

Airborne carcinogens, including tobacco smoke, are strongly implicated in the etiology of both squamous cell carcinomas of the head and neck and squamous cell carcinoma of the lung (1). Similar to the case for all head and neck cancers (HNC), squamous cell carcinoma is the most common histologic subtype of oral cancer (OSCC), accounting for nearly $90 \%$ of all cases of oral cancer (2). OSCC ranks sixth in cancer incidence worldwide, with approximately 300,400 newly diagnosed cases and 145,400 deaths each year, and the cancer is often diagnosed at an advanced stage $(3,4)$. The two most important risk factors for OSCC, accounting for over $80 \%$ of all patients, are tobacco and alcohol (5). Smoking is also a strong risk factor for lung squamous cell carcinoma (LSCC), which accounts for 15 to $30 \%$ of all cases of lung cancer; a trend towards decrease in the incidence rates associated with a decrease in smoking rates has been reported in developed countries (6). Due to the occurrence of lung cancer in epidemic proportions worldwide, however, urgent development of countermeasures is still required for LSCC.

Recent advances in molecular-targeted therapies have brought about significant improvements in the treatment outcomes of some advanced cancers (7). Especially, tyrosine-kinase inhibitors (TKIs) have proven to be significantly beneficial for patients with advanced adenocarcinomas of the lung harboring driver oncogene mutations, including activating epidermal growth factor receptor $(E G F R)$ mutations and rearrangements of the anaplastic lymphoma kinase gene (8). On the other hand, in the case of LSCC, no driver mutations or effective TKIs have been identified yet, thus no effective molecular-targeted therapies against this cancer are available to date. Although cetuximab, an anti-EGFR antibody, administered in combination with radiation (9) or cytotoxic chemotherapy (10) has been revealed to be effective for advanced $\mathrm{HNC}$, including OSCC, and another anti-EGFR antibody, necitumumab, administered in combination with cytotoxic chemotherapy (11) has been revealed to be effective for advanced LSCC, most patients with advanced OSCC or LSCC eventually exhibit disease progression. Therefore, novel molecular targets must be sought for developing novel molecular-targeted therapies for OSCC and LSCC. 
The epithelial stromal interaction 1 gene (EPSTII) is a gene mapped to human chromosome 13q13.3, that has received increasing attention in recent years. It has been revealed to be expressed in some normal tissues, including the spleen, small intestine, salivary glands, testes, germinal centers of lymph nodes, and placenta (12-15). In addition, overexpression of the gene has been reported in primary cancers of the breast, colorectal and pancreas $(12,16,17)$. In breast cancers, high expression levels of EPSTII have been revealed to be strongly associated with enhanced tumor cell migration ability and tumor invasiveness, a high propensity for metastasis, and escape from apoptosis, suggesting that EPSTI1 expression may be an independent prognostic marker in patients with breast cancer $(13,18,19)$. Furthermore, accumulated evidence has revealed multiple roles of EPSTI1 in the immune response; it has been demonstrated to be associated with the establishment of immune privilege, development of autoimmune diseases, control of viral infections, and activation of macrophages. The gene has been revealed to have a role in the immune privilege of the testes (20), and a genome-wide association study has linked the gene to male fertility (21). Overexpression of EPSTII has been reported in the peripheral blood cells of patients with systemic lupus erythematosus (22), and such overexpression was revealed to be associated with abnormal B-cell activation via the $\mathrm{NF}-\kappa \mathrm{B}$ signaling pathway in primary Sjogren syndrome (23); expression of the gene induced by $\mathrm{HCV}$ infection has been revealed to effectively inhibit viral replication and exert antiviral effects through upregulation of IL-28A (24). Finally, the gene has also been revealed to play a role in modulating macrophage activation and polarization (15). However, despite these already known functions of EPSTI1, its roles in human OSCC and LSCC remain unclear.

\section{Materials and methods}

Cell lines. A human normal oral keratinocyte cell line, HNOKs, derived from a mixture of healthy gingival specimens of 22- to 35-year-old patients, was established and maintained as previously described (25). Human OSCC cell lines HSC2, HSC3, and HSC4 were purchased from RIKEN Bioresource Center, and HSC3-M3 was purchased from the Human Science Resources Bank. The cells were cultured in DMEM (Sigma-Aldrich; Merck KGaA). A normal human lung cell line, BEAS-2B, and the LSCC cell lines LK-2, EBC-1 and H226 were purchased from JCRB cell bank. BEAS-2B, LK-2 and H226 were cultured in PRMI-1640 medium, and EBC-1 was cultured in MEM (both from Sigma-Aldrich; Merck KGaA). All the culture media were supplemented with $10 \%$ fetal bovine serum and $1 \%$ antibiotics, including penicillin and streptomycin (all from Life Technologies; Thermo Fisher Scientific, Inc.). All the cell lines were cultured in a humidified incubator at $37^{\circ} \mathrm{C}$ in a $5 \%$ $\mathrm{CO}_{2}$ atmosphere.

Expression of $m R N A$ and protein. The cells were washed three times with phosphate-buffered saline (PBS), followed by extraction of total RNA and protein. The mRNA expression levels were quantified by real-time reverse transcriptionquantitative polymerase chain reaction (RT-qPCR) with
SYBR green expression assays (Roche Diagnostics), in accordance with the manufacturer's instructions. In brief, total RNA was extracted using TRIzol reagent (Invitrogen; Thermo Fisher Scientific, Inc.) when the cells reached $80 \%$ confluence in the $10-\mathrm{cm}$ culture plates. Reverse transcription was performed using the ReverTra Ace qPCR RT Master Mix (Toyobo Life Science). Gene expression was measured using LightCycler 480 Instrument (Roche Diagnostics). The thermocycling conditions for the PCR were as follows: initial denaturation at $95^{\circ} \mathrm{C}$ for $10 \mathrm{~min}$, followed by 45 cycles of amplification at $95^{\circ} \mathrm{C}(10 \mathrm{sec})$ for denaturation, $60^{\circ} \mathrm{C}(10 \mathrm{sec})$ for annealing and $72^{\circ} \mathrm{C}$ for extension, followed by a cooling step at $40^{\circ} \mathrm{C}$ for $30 \mathrm{sec}$. GAPDH was used as the internal control. Transcript amounts were estimated from respective standard curves and normalized to GAPDH. Primers were designed using the Universal Probe Library Assay Design Center (http://lifescience.roche.com/). The primers for EPSTII were 5'-CCGGAGAAATGAGATACAAAGAAT-3' (forward) and 5'-GGTGAACCGGTTTAGCTCTG -3' (reverse), and the primers for $G A P D H$ were 5'-AACATCATCCCTGCCTCT ACTGG-3' (forward) and 5'-TTGAAGTCAGAGGAGACC ACTG-3' (reverse).

For the evaluation of the protein expression, the cells were washed twice with cold phosphate-buffered saline (PBS) and collected with lysis buffer [7 $\mathrm{M}$ urea, 2M thiourea, 4\% (w/v) CHAPS, and $10 \mathrm{mM}$ Tris] with a proteinase inhibitor cocktail (Roche Diagnostics). The protein concentration was first assessed by the Bradford method, followed by western blot analysis. Protein extracts $(20 \mu \mathrm{g} /$ lane $)$ were separated by a $4-12 \%$ Bis-Tris gel (Thermo Fisher Scientific, Inc.), and transferred to nitrocellulose membranes. Subsequently, the membrane was blocked for $1 \mathrm{~h}$ at room temperature in $5 \%$ skim milk, and then incubated with primary antibodies $(1: 1,000)$ overnight at $4^{\circ} \mathrm{C}$. The membranes were washed with $0.1 \%$ Tween-20 in Tris-buffered saline, three times $(15 \mathrm{~min}$ for each wash), followed by incubation with a secondary antibody with anti-mouse (cat. no. S3721) or anti-rabbit IgG (cat. no. S3731) (1:2,000; Promega Corporation) for $1.5 \mathrm{~h}$ at room temperature. Finally, the bands were detected using clarity Western ECL Substrate (Bio-Rad Laboratories, Inc.), and immunoblotting images were visualized by exposing the membranes to the BioRad ChemiDoc ${ }^{\mathrm{TM}}$ XRS System (BioRad Laboratories, Inc.). The densitometric analysis of the protein expression was performed using Image Lab software 6.0.1 (Bio Rad Laboratories, Inc.).

The antibodies used were mouse anti-EPSTI1 monoclonal antibody (cat. no. AT1934a; Abcepta; Abgent, Inc.), rabbit anti-E-cadherin (product no. 3195), anti-N-cadherin (product no. 13116), anti-p21 (product no. 2947), anti-cyclin D1 (product no. 55506), and anti-CDK2 (product no. 18048) monoclonal antibodies (all from Cell Signaling Technology, Inc.), and mouse anti-fibronectin (product no. SAB4200760), anti- $\beta$-actin (product no. SAB1305567) (both from Sigma Aldrich; Merck KGaA), and anti- $\alpha$-tubulin monoclonal antibodies (cat. no. sc-5286; Santa Cruz Biotechnology, Inc.).

Transfection of plasmids. OSCC-derived cells (HSC3-M3 and HSC4) were transfected with EPSTI1-suppressing shRNA or the control plasmid, shMock (cat. no. sc-105335-SH and cat. no. sc-108060, respectively, Santa Cruz Biotechnology, 
Inc.), at a concentration of $2.5 \mu \mathrm{g} /$ well, using Lipofectamine 3000 (Thermo Fisher Scientific, Inc.), and then incubated overnight at $37^{\circ} \mathrm{C}$. After the transfection, the cells exhibiting stable transfection were selected by taking advantage of the selection marker and its inhibitor, puromycin (Santa Cruz Biotechnology, Inc.). LSCC-derived cells (LK-2 and EBC-1) were transfected with EPSTI1-overexpressing plasmid (OriGene Technologies, Inc.) or the control plasmid (OriGene Technologies, Inc.) at a concentration of $2.5 \mu \mathrm{g} /$ well, using Lipofectamine 3000 and incubated overnight at $37^{\circ} \mathrm{C}$. Following the transfection, the cells exhibiting stable transfection were selected by utilizing the selection marker and its inhibitor G-418 Solution (Roche Diagnostics). Then, incubation of the selected cells with the inhibitors for 2 to 3 weeks yielded individual clones from each of the EPSTI1-suppressed OSCC cell lines and EPSTI1-overexpressing LSCC cell lines; these clones and the control cell lines were used for further experiments.

Proliferation assay. To evaluate the cell proliferation ability, shEPSTI1 and shMock cells (HSC3-M3 and HSC4), oeEPSTI1 and OeMock cells $\left(5 \times 10^{4}\right.$ cells/plate) were seeded on to a $6-\mathrm{cm}$ plate, and the number of cells were counted every $24 \mathrm{~h}$ in triplicate using a hemocytometer to measure the chronological changes.

Wound healing assay. For evaluating the cell migration ability, $2 \times 10^{5}$ cells/well were seeded on to a 6 -well plate overnight until they reached $95 \%$ confluence; the surface of the plate was then scratched with the tip of a 1,000- $\mu 1$ micropipette and the culture medium was immediately washed off with PBS; the cells were then cultured in serum-free medium for a further $24 \mathrm{~h}$. The scratch scars were viewed under an inverted microscope (x10 magnification of objective) (ECLIPSE TS100; Nikon Corporation), and the scratched areas were quantified using the Lenaraf220b free software (available at http://www. vector.co.jp/soft/dl/win95/art/se312811.html). The wound closure rates were determined as a percentage of the total repaired area per hour and normalized to the control.

Cell cycle analysis. Cells were harvested with trypsin and centrifuged for $5 \mathrm{~min}$ at $300 \mathrm{x} \mathrm{g}$ at room temperature, and then the concentration was adjusted to $1.0 \times 10^{6}$ cells $/ \mathrm{ml}$. Cell cycle analyses were performed using the Cycletest ${ }^{\mathrm{TM}}$ Plus DNA reagent kit (BD Biosciences) and the BD Accuri C6 Flow Cytometer (BD Biosciences), in accordance with the manufacturers' instructions. For data analysis FCS Express 4 (De Novo Software) was used.

$P C R$ array. Differences in the mRNA expression of the EPSTI1-related genes in the two EPSTI1-downregulated OSCC cell lines, HSC3-M3 and HSC4, and two EPSTI1-overexpressing LSCC cell lines, LK2 and EBC-1, as compared to the expression levels in the corresponding parental cells, were detected using the $\mathrm{RT}^{2}$ Profiler PCR Array Human Cancer Pathway Finder (cat. no. PAHS-033ZF-6; Qiagen, Inc.). In brief, cDNA was synthesized from the total RNA extracted from each cell line using the RT ${ }^{2}$ First Strand Kit (Qiagen, Inc.). RT ${ }^{2}$ SYBR Green qPCR Master Mix (Qiagen, Inc.) was used for the PCR arrays, in accordance with the manufacturer's instructions.
Bioinformatics analysis. The gene expression in clinical samples was obtained from the Oncomine Platform (http://www.oncomine.org).

Statistical analysis. The unpaired Student's t-test was used to analyze the statistical significance of differences between two groups. One-way ANOVA with Dunnett's post hoc test calculated by GraphPad Prism software (version 8; GraphPad software, Inc.) were used to evaluate the differences of multiple comparisons. All experiments were performed in triplicate and repeated 3 times, unless otherwise specified. $\mathrm{P}<0.05$ was considered to indicate statistically significant differences. The data are expressed as the means \pm standard error (SE).

\section{Results}

EPSTII expression in the OSCC and LSCC cell lines. The series of RT-qPCR and western blot analyses in the four OSCC (HSC2, HSC3, HSC3-M3 and HSC4) and three LSCC (EBC-1, LK-2 and H226) cell lines revealed significant overexpression of EPSTI1 in all of the OSCC cell lines (Fig. 1A and C), except for protein expression in HSC2, and significantly suppressed expression of the gene in all of the LSCC cell lines (with the exception of EPST11 mRNA expression in the LK-2 cell line) (Fig. 1B and D), as compared to the expression levels in the corresponding control cell lines, HNOKs and BEAS-2B, respectively (Fig. 1B).

Establishment of cells with EPSTI1 knockdown and EPST11 overexpression. Based on the results of the experiments aforementioned (Fig. 1C and D), the OSCC cell lines, HSC3-M3 and HSC4, and LSCC cell lines, LK-2 and EBC-1, were selected for further experiments because the former exhibited the strongest expression, among all the OSCC cell lines, of the EPSTI1 protein, and the latter exhibited the weakest expression, among all the LSCC cell lines, of the EPSTI1 protein. HSC3-M3 and HSC4, were transfected with EPSTI1 shRNA plasmid to suppress the gene expression, which yielded two clones for each cell line, whereas LK-2 and EBC-1 were transfected with the EPSTI1 overexpression plasmid, which yielded a single clone per cell line, to further investigate the roles of the gene. As transfection of the EPSTI1 overexpression plasmid into the LSCC cell lines was somewhat difficult, only a single clone was obtained for each cell line. The alterations in the expression levels of the gene were verified by RT-qPCR and western blot analysis. The results revealed significant downregulation of EPSTI1 at the mRNA and/or protein level in the shRNA-transfected OSCC cell lines as compared to the shMock-transfected cells (Fig. 2A and C); similarly, significant upregulation of the gene at the mRNA and/or protein level was confirmed in the LSCC cells transfected with the overexpression vector as compared to the oeMock-transfected cells (Fig. 2B and D).

Altered expression of EPSTI1 induces alterations in the cell proliferation ability and cell cycle progression. Cell propagation was significantly suppressed by downregulation of EPSTI1 in the two OSCC cell lines (Fig. 3A), and by upregulation of the gene in the two LSCC cell lines (Fig. 3B). Cell cycle analysis demonstrated accumulation of cells in the G1 

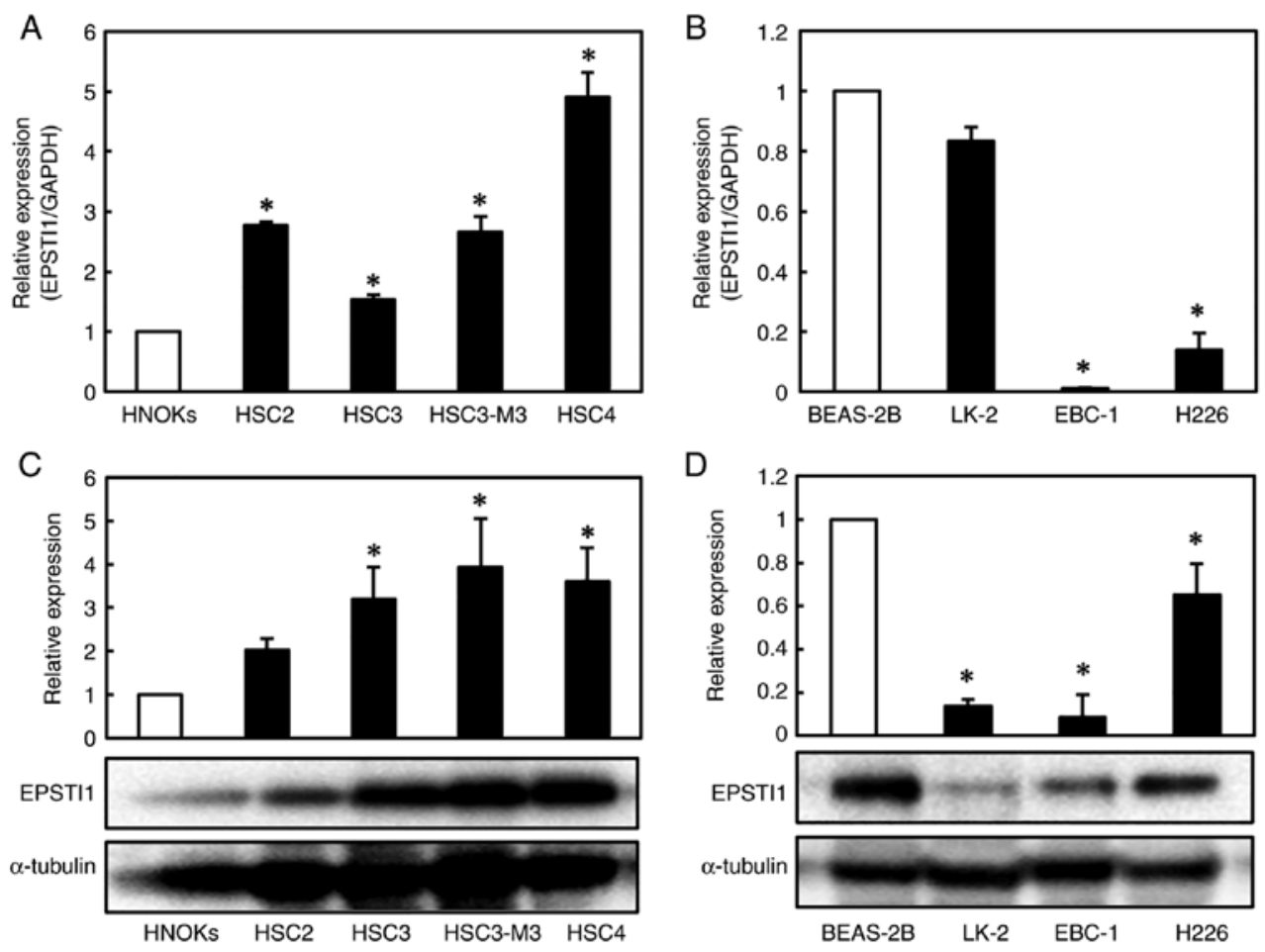

Figure 1. Expression of EPSTI1 in human OSCC and LSCC cell lines. Quantification of EPSTI1 mRNA expression levels by RT-qPCR revealed (A) significantly increased expression levels in the four human OSCC cell lines (HSC2, HSC3, HSC3-M3 and HSC4) as compared to the levels in the control cell line, HNOKs, and (B) significantly reduced expression levels in two of the three human LSCC cell lines (EBC-1 and H226, but not LK-2) as compared to the levels in the normal control cell line, BEAS-2B. Western blotting, using a-tubulin as the internal control, confirmed (C) significant upregulation of the gene in the OSCC cell lines and (D) significant downregulation of the gene in the LSCC cell lines, including LK-2. The values on the y-axes represent the ratios to the expression levels in the normal control cell lines. Each experiment was performed in triplicate and repeated three times, and data are presented as the means \pm SE. "P<0.05. EPSTI, epithelial-stromal interaction 1; OSCC, oral squamous cell carcinoma; LSCC, lung squamous cell carcinoma; SE, standard error.
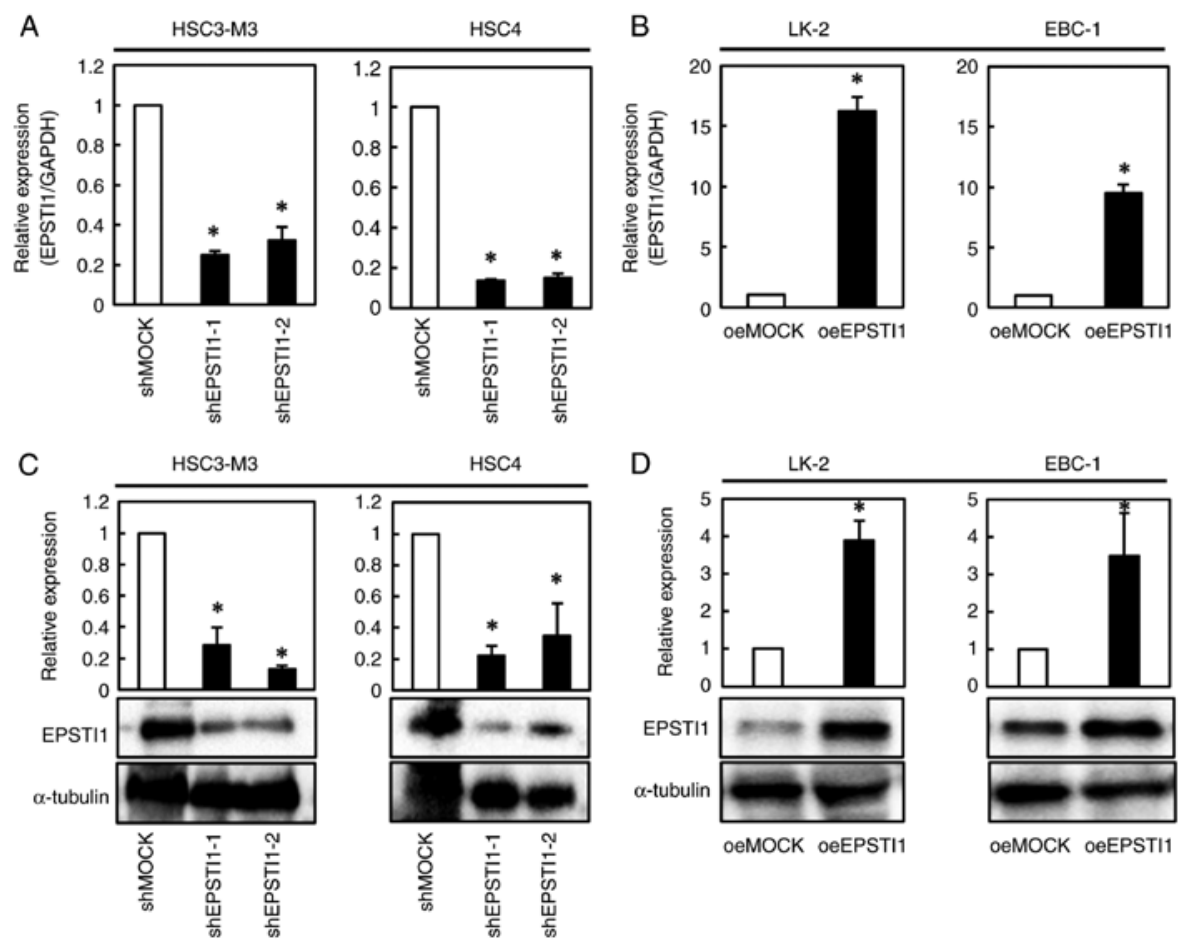

Figure 2. Confirmation of the modified gene expression of EPSTII in the OSCC and LSCC cell lines. For further elucidation of the functions of EPSTII, expression of the gene was knocked down in two OSCC cell lines, HSC3-M3 and HSC4, by transfection of shRNA, and overexpressed in two LSCC cell lines, LK-2 and EBC-1, using overexpressing vectors. Consequently, EPST11 mRNA expression was (A) significantly suppressed in the two OSCC cells as compared to that in the mock transfectants, and (B) significantly overexpressed in the two LSCC cells as compared to that in the mock-transfected cells. In addition, western blotting confirmed (C) reduced EPSTI1 protein expression in the shRNA-transfected OSCC cell lines, and (D) overexpression of EPST11 protein in the overexpressing vector-transfected LSCC cell lines. Each experiment was performed in triplicate and repeated three times, and data are presented as the means $\pm \mathrm{SE} .{ }^{*} \mathrm{P}<0.05$. EPSTI, epithelial-stromal interaction 1; OSCC, oral squamous cell carcinoma; LSCC, lung squamous cell carcinoma; SE, standard error. 
A

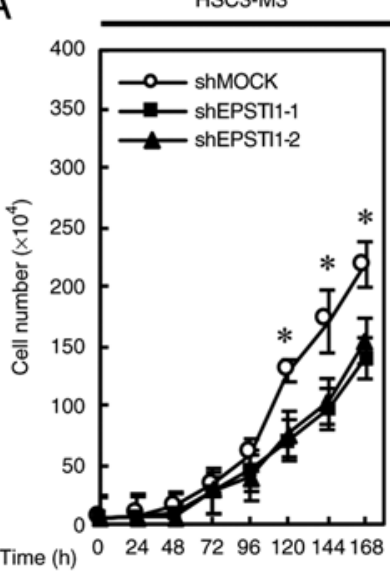

HSC4

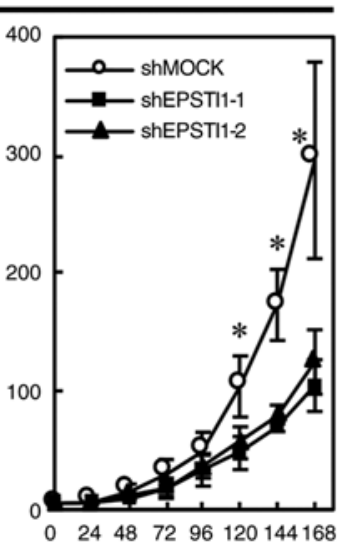

B

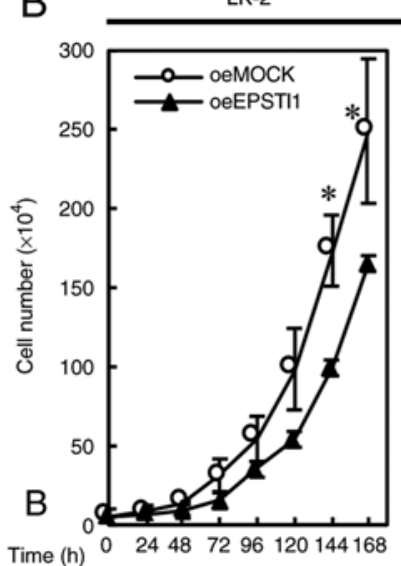

EBC-1

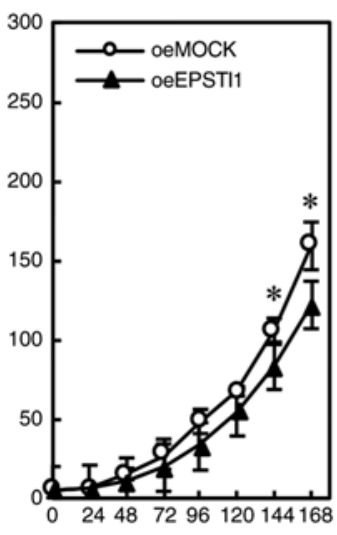

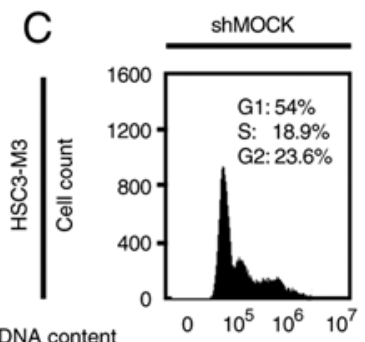
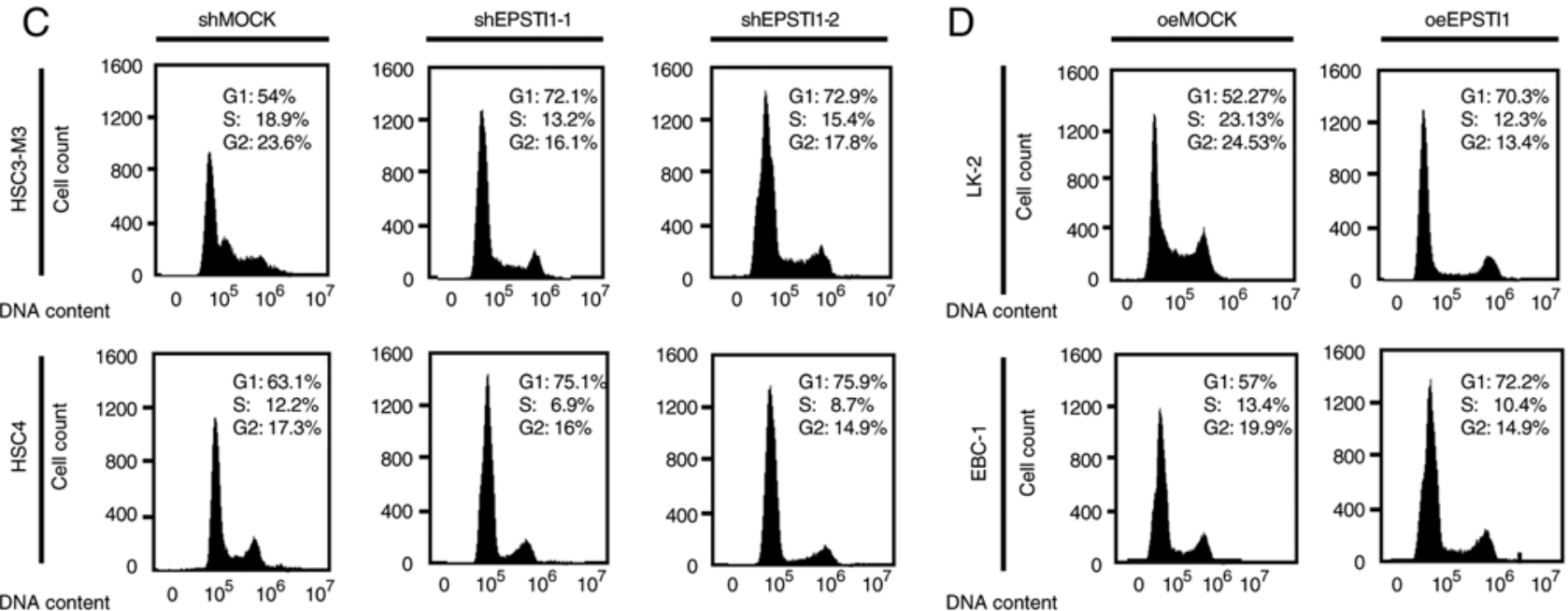

$\mathrm{E}$

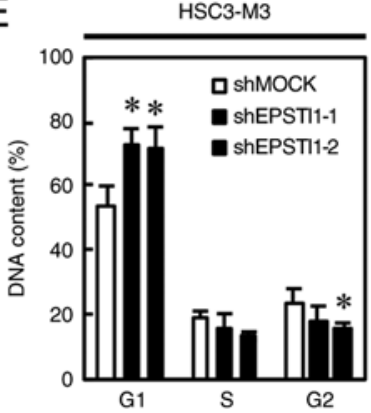

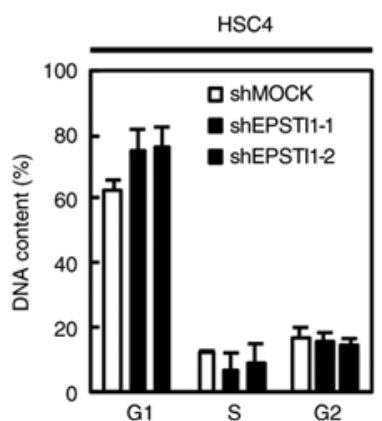

$\mathrm{F}$
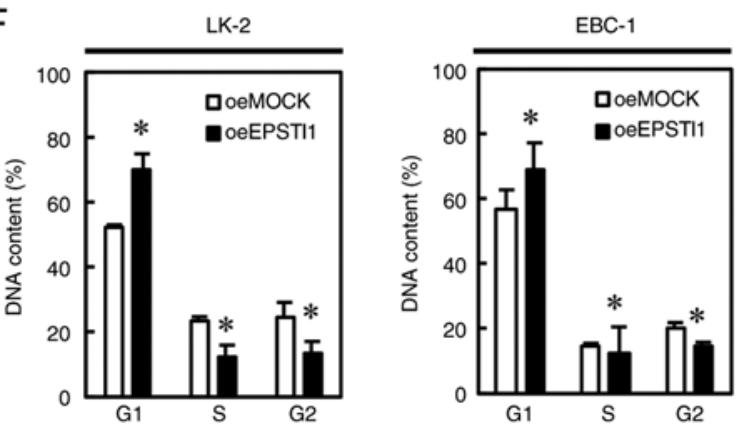

Figure 3. Inhibition of cell proliferation and induction of cell cycle arrest in the G1 phase is associated with modified EPSTI1 expression. Genetically modified expression of EPSTII; (A) downregulation of the gene in two OSCC cell lines was associated with significantly suppressed cell proliferation and ( $\mathrm{C}$ and $\mathrm{E}$ ) increase in the G1 population, although statistical significance was marginal in HSC4 ( $\mathrm{P}=0.0533)$, suggesting cell cycle arrest. Similarly, (B) overexpression of the gene in two LSCC cell lines significantly suppressed cell proliferation and (D and F) induced cell cycle arrest in the G1 phase, with a significant decrease in the population of cells in the S and G2 phases in the LK-2 cell line, and in the population of cells in the G2 phase in the EBC-1 cell line. Each experiment was performed in triplicate and repeated six times for A and B, and three times for C-F. Data are presented as the means \pm SE. " $\mathrm{P}<0.05$. EPSTI, epithelial-stromal interaction 1; OSCC, oral squamous cell carcinoma; LSCC, lung squamous cell carcinoma; SE, standard error.

phase in every OSCC cell line with downregulated EPSTII expression and every LSCC cell line with upregulated EPSTII expression with statistical significance except for HSC4 which revealed a trend of $\mathrm{G} 1$ accumulation $(\mathrm{P}=0.053)$ (Fig. 3C-F). To confirm this finding, the expression levels of the cell cycle-related genes, $p 21, C D K 2$, and cyclin D1, were investigated. As anticipated, upregulation of $p 21$ and downregulation of $C D K 2$ and cyclin D1 were observed in every OSCC cell line with downregulated EPSTII expression (Fig. 4A and C) and in every LSCC cell line with upregulated EPSTII expression
(Fig. 4B and D), although statistical significance was marginal for $\mathrm{p} 21(\mathrm{P}=0.08)$ and cyclin $\mathrm{D} 1(\mathrm{P}=0.06)$ in HSC3-M3 cells.

Altered expression of EPSTI1 induces altered cell migration ability and epithelial-mesenchymal transition. The wound healing assay revealed significantly decreased cell migration ability in OSCC cell lines with downregulated EPSTII expression, HSC3-M3 and HSC4 (Fig. 5A) and in the LSCC cell line EBC-1 with upregulated EPSTII expression (LK-2 was not used since the cells were scattered once the cells reached 

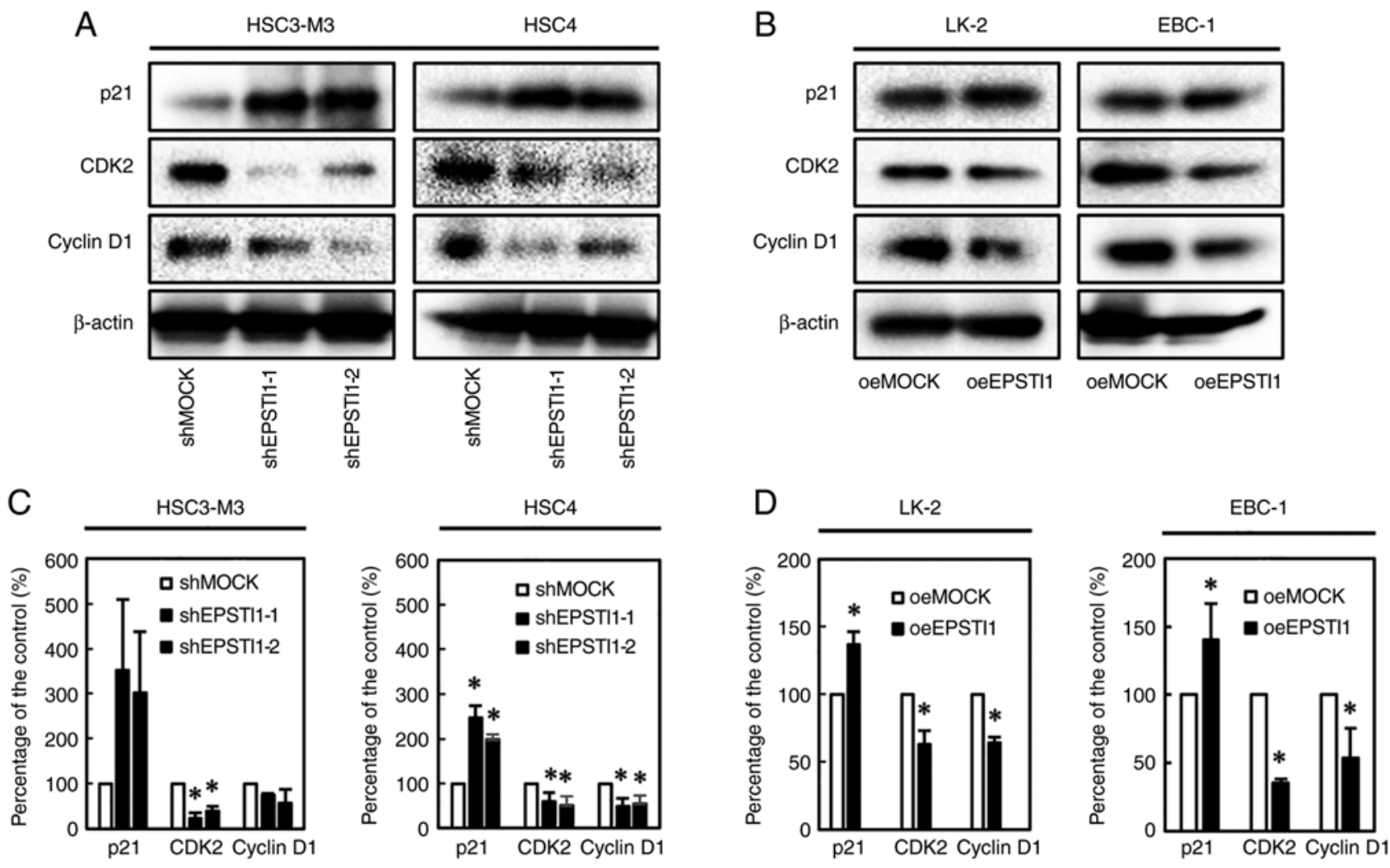

Figure 4. Expression of the cell-cycle-related genes, $p 21, C D K 2$, and Cyclin D1. Western blotting revealed enhanced expression of $p 21$, and suppressed expression of CDK2 and Cyclin D1 associated with modified EPSTI1 expression (upregulation and downregulation, respectively) in the (A and C) OSCC and (B and D) LSCC cell lines, although statistical significance was marginal for $\mathrm{p} 21(\mathrm{P}=0.08)$ and cyclin $\mathrm{D} 1(\mathrm{P}=0.06)$ in HSC3-M3, which appears consistent with cell cycle arrest in each of the cell lines. Each experiment was performed in triplicate and repeated three times, and data are presented as the means \pm SE. ${ }^{*} \mathrm{P}<0.05$. EPSTI, epithelial-stromal interaction 1; OSCC, oral squamous cell carcinoma; LSCC, lung squamous cell carcinoma; SE, standard error.

confluence) (Fig. 5B). The expression levels of the epithelial-mesenchymal transition (EMT)-related proteins were evaluated by western blot analysis. Significant upregulation of E-cadherin and downregulation of N-cadherin and fibronectin, suggestive of a suppressed EMT phenotype, were observed in OSCC cell line with downregulated EPSTII expression and LSCC cell line with upregulated EPSTII expression with exception in fibronectin in HSC4 which exhibited marginal statistical significance $(\mathrm{P}=0.08)$ (Fig. 5C-F).

EPSTI1-related genes as shown by PCR arrays. PCR arrays identified some upstream and downstream genes in relation to the altered EPSTII expression in the two OSCC and two LSCC cell lines, as summarized in Tables I and II. Overexpression of carbonic anhydrase 9 (CA9) and downregulation of C-C motif chemokine ligand 2 (CCL2) were identified in both the EPSTI1-suppressed OSCC cell lines, as compared to the corresponding control cell line (Table I), whereas downregulation of cyclin D2 (CCND2), baculoviral IAP repeat containing 3 (BIRC3) and insulin-like growth factor binding protein 7 (IGFBP7) were identified in all the EPSTI1-overexpressing LSCC cell lines, as compared to the corresponding control cell line (Table II).

\section{Discussion}

The present study revealed significantly higher expression levels of ESPTII in all the four OSCC cell lines examined in the present study as compared to the corresponding control cell line, and significantly lower expression levels of the EPSTII gene in all the three LSCC cell lines examined as compared to the corresponding control cell line. Both OSCC cell lines with forced downregulation of the gene and LSCC cell lines with overexpression of the gene exhibited significantly suppressed tumor cell proliferation ability. Analyses of the cell cycle and of genes related to the cell cycle, i.e., $p 21, C D K 2$ and cyclin D1, generally supported the finding of cell cycle arrest in the G1 phase in both the aforementioned OSCC and LSCC cell lines. OSCC cell lines with downregulation of EPSTII and LSCC cell lines with upregulation of EPSTII similarly revealed suppressed EMT, as assessed by the cell migration activity and cellular expression levels of E-cadherin, $\mathrm{N}$-cadherin and fibronectin. Therefore, EPSTII was demonstrated to play roles in enhancing the malignant phenotypes, including the cell proliferation ability and EMT in OSCC, whereas it played the opposite roles in LSCC.

Overexpression of EPSTII was first identified in breast cancer, and overexpression of the gene is considered to drive the malignant phenotype, including the cell proliferation and migration abilities, invasiveness, EMT, and escape from apoptosis $(12-14,18,19)$. It has also been identified as being upregulated in cell lines derived from peritoneal disseminations of colorectal cancer as compared to the cell lines derived from primary colorectal cancers (16). In pancreatic cancer, gene expression was associated with resistance to therapy with an oncolytic virus (17). 
A
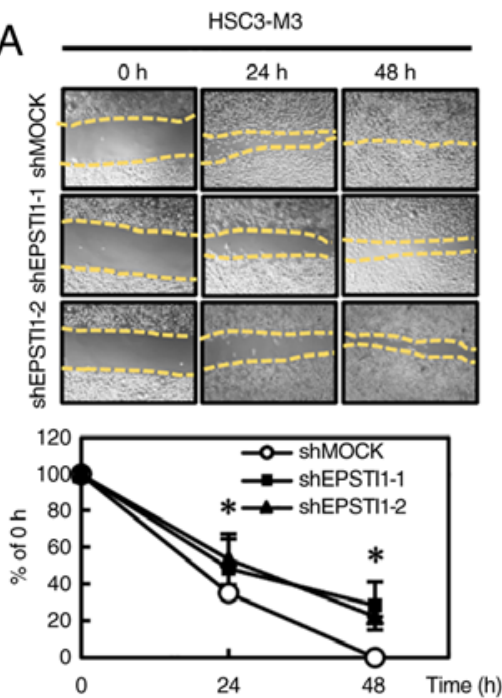

C

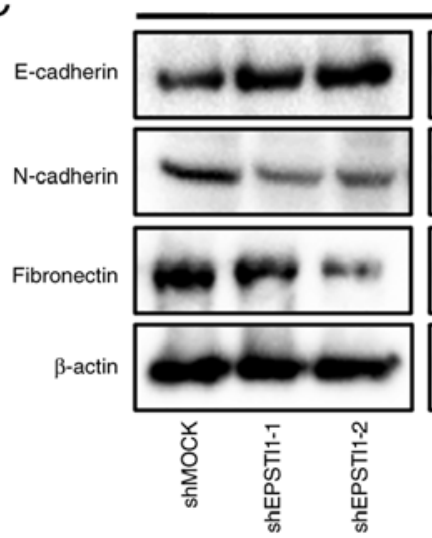

E

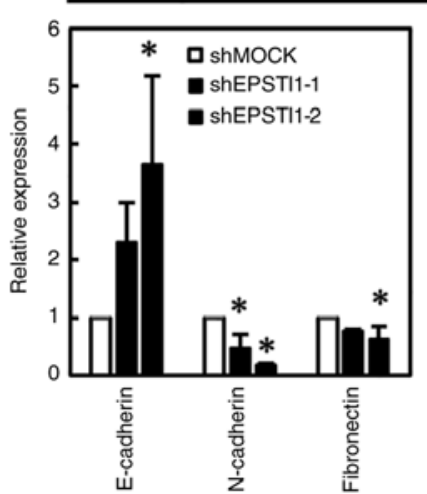

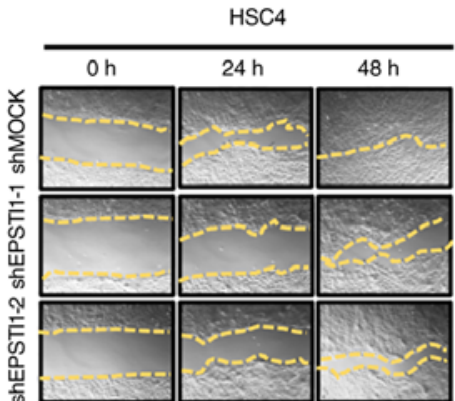

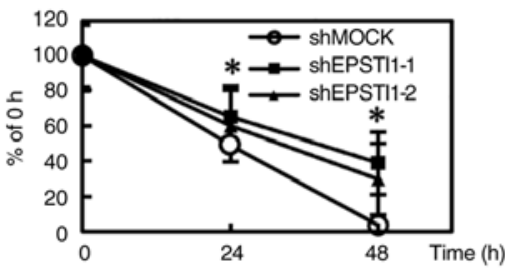

B

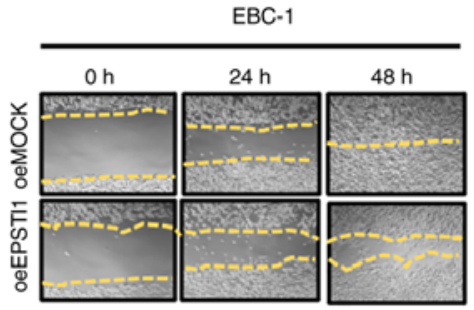

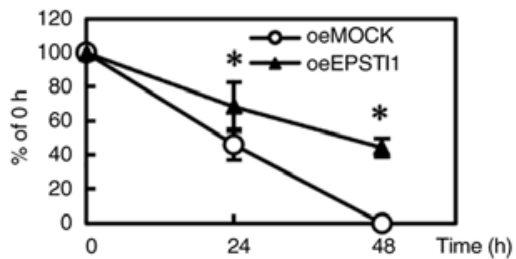

D

LK-2 EBC-1
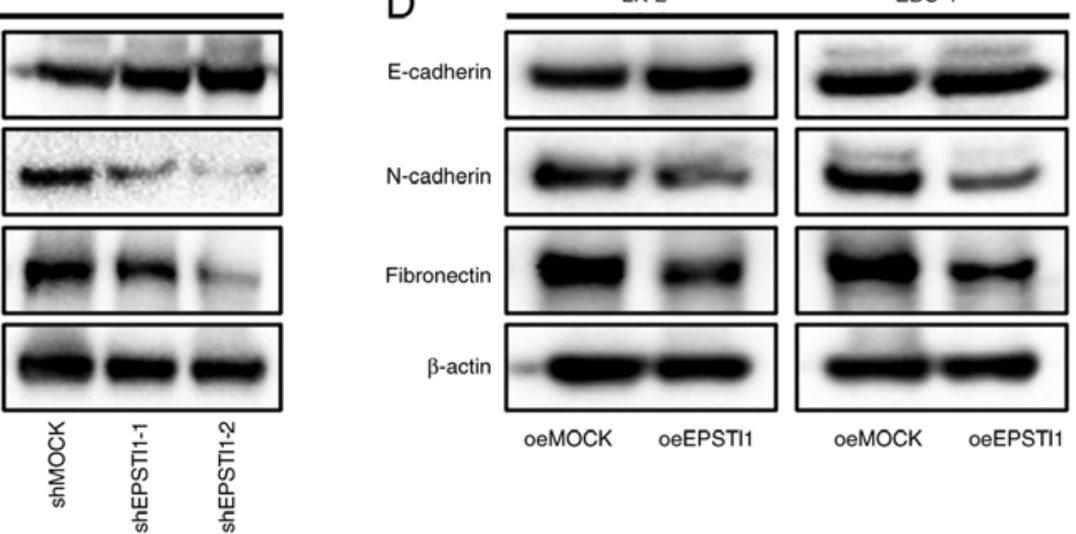

F

LK-2

EBC-1
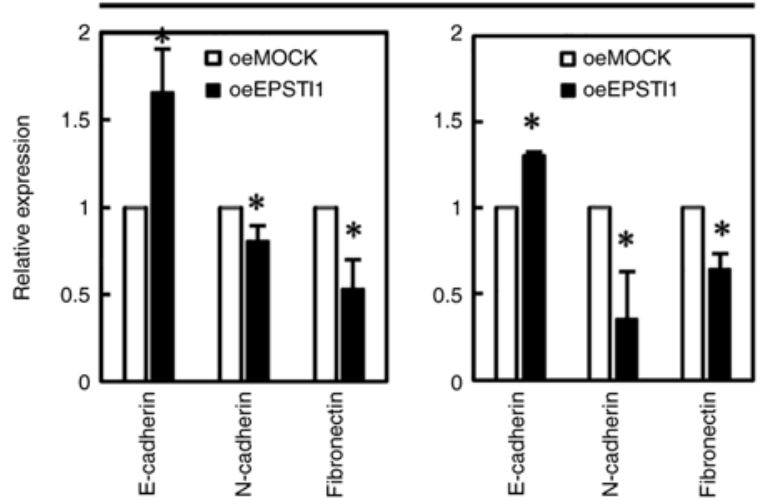

Figure 5. Decreased cell migration capability and reversal of the EMT phenotype in tumor cells with modified EPSTI1 expression. (A) Downregulation of EPSTI1 in OSCC and (B) upregulation of the gene in LSCC were associated with significant suppression of the cell migration ability, suggesting reversal of the EMT phenotype. Accordingly, (C and E) downregulation of EPSTI1 in OSCC and (D and F) upregulation of the gene in LSCC were also associated with significantly increased expression levels of E-cadherin and significantly suppressed expression levels of N-cadherin and fibronectin with exception in fibronectin in HSC4 which exhibited marginal statistical significance $(\mathrm{P}=0.08)$, again suggesting reversal of the EMT phenotype. The cell migration assay in LSCC was performed using only EBC-1, but not LK-2. Each experiment was performed in triplicate and repeated three times, and data are presented as the means \pm SE. ${ }^{*} \mathrm{P}<0.05$. EMT, epithelial-mesenchymal transition; EPSTI, epithelial-stromal interaction 1; OSCC, oral squamous cell carcinoma; LSCC, lung squamous cell carcinoma; SE, standard error.

In the present study, EPSTII was demonstrated to enhance the malignant phenotype in OSCC, similar to breast $(13,14,18,19)$ and colorectal cancers $(16)$; notably, the gene played the opposite role in the LCSS cell lines. The gene expression in clinical samples obtained from the Oncomine database revealed higher expression levels of the gene in cancer tissues as compared to normal tissues (26). Unfortunately, relevant data could not be obtained in the Oncomine database regarding EPSTI1 expression in LSCC (27). Although the contrasting roles of the same gene in different cancers, i.e., OSCC and LSCC, appear somewhat unusual, a few similar examples have been reported previously. Specifically, a T-box 
Table I. Altered gene expression in OSCC with EPSTI1 knockdown as assessed by PCR array.

\begin{tabular}{|c|c|c|c|}
\hline \multicolumn{2}{|c|}{ HSC3-M3 } & \multicolumn{2}{|c|}{ HSC4 } \\
\hline \multicolumn{2}{|c|}{ Gene symbol $^{\mathrm{a}}\left(\right.$ ratio $\left.^{\mathrm{b}}\right)$} & \multicolumn{2}{|c|}{ Gene symbol (ratio) } \\
\hline Upregulated & Downregulated & Upregulated & Downregulated \\
\hline$\underline{\operatorname{CA9}}(7.2)^{\mathrm{c}}$ & $\underline{\text { CCL2 }}(-3.4)$ & LDHA (79.9) & XIAP (-224.9) \\
\hline SERPINF1 (4.2) & BIRC3 (-2.5) & MKI67 (15.0) & IGFBP3 (-38.5) \\
\hline FGF2 (3.5) & SERPINB2 (-2.4) & SERPINB2 (14.7) & HMOX1 (-35.7) \\
\hline TBX2 (3.0) & & BMI1 (7.9) & $\underline{\text { CCL2 }(-26.5)}$ \\
\hline PGF (2.9) & & PINX1 (5.8) & TBX2 (-8.3) \\
\hline \multirow[t]{18}{*}{ DDIT3 (2.1) } & & DKC1 (4.3) & DDIT3 (-7.3) \\
\hline & & CA9 (3.9) & FLT1 $(-5.8)$ \\
\hline & & WEE1 (2.5) & SOX10 $(-5.8)$ \\
\hline & & LIG4 (2.4) & TEK (-5.8) \\
\hline & & AURKA (2.4) & EPO (-5.4) \\
\hline & & APAF1 (2.3) & IGFBP5 (-5.4) \\
\hline & & VEGFC (2.3) & LPL (-5.2) \\
\hline & & MAPK14 (2.1) & PPP1R15A (-4.6) \\
\hline & & CDH2 (2.1) & GADD45G (-3.4) \\
\hline & & CDC20 (2.0) & COX5A $(-3.3)$ \\
\hline & & & CASP7 (-3.1) \\
\hline & & & GSC (-2.9) \\
\hline & & & G6PD (-2.3) \\
\hline & & & ANGPT2 (-2.2) \\
\hline & & & KDR (-2.2) \\
\hline & & & CASP9 (-2.2) \\
\hline & & & TINF2 (-2.1) \\
\hline & & & SNAI1 (-2.1) \\
\hline
\end{tabular}

a'Genes up- or downregulated by more than 2-fold are listed. ${ }^{b}$ Each experiment was carried out in triplicate, and the mean ratios are presented in parentheses. ${ }^{c}$ Gene symbols in bold font and underlined are those that were common to the 2 cell lines. EPSTI, epithelial-stromal interaction 1; OSCC, oral squamous cell carcinoma; PCR, polymerase chain reaction.

family member, $T B X 2$, was reported to be amplified and overexpressed in breast cancer (28) and its overexpression has been revealed to be associated with a high pathological grade in prostatic cancer (29); conversely, the gene has been revealed to be downregulated in non-small cell lung cancer (30); although further details have not yet been elucidated. Therefore, it is considered that the present study findings, provide further insight into understanding the multi-functional roles of EPSTI1.

To elucidate the mechanisms underlying these phenomena, a series of PCR array assays were performed. Upregulation of the CA9 gene, observed in both the OSCC cell lines with forced downregulation of EPSTII expression, has been revealed to augment the cell proliferation and transformation ability under hypoxic conditions, under the regulation of the transcription factor HIF-1 $\alpha$ (31). Downregulation of the CCL2 gene, a member of the chemokine superfamily, observed in the same cell lines, has been demonstrated to promote cancer progression through recruiting inflammatory cells in multiple cancers (32-34), and to promote EMT-associated cell migration ability in OSCC (35). Conversely, downregulation of $C C N D 2, B I R C 3$, and $I G F B P 7$, observed in both the EPSTI1-overexpressing LSCC cell lines, has been revealed to promote $\mathrm{G} 1 / \mathrm{S}$ phase transition (CCND2) (36), suppress apoptosis by inhibiting caspase-3 (BIRC3) (37), and to promote TGF-b-induced EMT in human renal proximal tubular epithelial cells (IGFBP7) (38). These studies, except for the information concerning $C A 9$, are all consistent with the phenomena observed in the OSCC and LSCC cell lines in the present study. There were other inconsistencies in the PCR array results, in addition to the case of $C A 9$. Although western blotting revealed downregulation of $\mathrm{N}$-cadherin in the HSC4 cell line with EPSTI1 knockdown, the PCR array disclosed upregulation of $\mathrm{CDH} 2$ in the same cell line. Since the results of western blot analysis are likely to be more reliable, comprehensive gene analysis by PCR array may be susceptible to error. $M K I 67$, as well as $C A 9$, revealed to be upregulated in the HSC4 cells with EPSTI1 knockdown, reportedly augment cell proliferation, whereas knockdown of EPSTII in the HSC4 suppressed cell proliferation in the present research. The finding that there were no commonly altered gene expression between the OSCC and LSCC cell lines may indicate that 
Table II. Altered gene expression in LSCC with EPSTI1 overexpression, as assessed by PCR array.

\begin{tabular}{|c|c|c|c|}
\hline \multicolumn{2}{|c|}{ LK-2 } & \multicolumn{2}{|c|}{ EBC-1 } \\
\hline \multicolumn{2}{|c|}{ Gene symbol $^{\mathrm{a}}\left(\right.$ ratio $\left.^{\mathrm{b}}\right)$} & \multicolumn{2}{|c|}{ Gene symbol (ratio) } \\
\hline Upregulated & Downregulated & Upregulated & Downregulated \\
\hline TBXT2 (28.0) & $\underline{\text { CCND2 }(-17.3)^{\mathrm{c}}}$ & HMOX1 (9.5) & CDC20 (-14.5) \\
\hline LPL (16.2) & SNAI2 (-11.3) & DDIT3 (9.5) & MKI67 (-11.1) \\
\hline IGFBP5 (5.3) & KRT14 (-6.2) & XIAP (3.4) & IGFBP7 (-10.7) \\
\hline CDH2 (3.9) & SERPINB2 (-4.1) & LIG4 (3.4) & $\operatorname{LPL}(-4.7)$ \\
\hline KDR (3.7) & SLC2A1 (-2.4) & CASP9 (3.0) & STMN1 (-4.6) \\
\hline HGDC (3.1) & $\underline{\operatorname{BIRC3}(-2.3)}$ & ARNT (2.9) & TEK (-4.0) \\
\hline SERPINF1 (3.1) & $\underline{\text { IGFBP7 (-2.2) }}$ & ERCC5 (2.7) & $\operatorname{CCL2}(-3.7)$ \\
\hline GSC (2.6) & ACSL4 (-2.1) & IGFBP3 (2.7) & AURKA (-3.6) \\
\hline GADD45G (2.4) & & PPP1R15A (2.6) & $\underline{\text { CCND3 (-2.9) }}$ \\
\hline SOD1 (2.2) & & SOX10 (2.2) & LDHA (-2.8) \\
\hline SNAL1 (2.1) & & TNKS (2.2) & CCND2 (-2.7) \\
\hline \multirow[t]{3}{*}{ ANGPT1 (2.1) } & & PINX1 (2.1) & PGF (-2.6) \\
\hline & & OCLN (2.0) & $\underline{\operatorname{BIRC3}(-2.3)}$ \\
\hline & & & SKP2 (-2.1) \\
\hline
\end{tabular}

${ }^{\mathrm{a}}$ Genes up- or downregulated by more than 2-fold are listed. ${ }^{\mathrm{b}}$ Each experiment was carried out in triplicate, and the mean ratios are presented in parentheses. ${ }^{\circ}$ Gene symbols in bold font and underlined are those that were common to the 2 cell lines. EPSTI, epithelial-stromal interaction 1 ; LSCC, lung squamous cell carcinoma; PCR, polymerase chain reaction.

EPSTII acts differently in the two cancers, which is also consistent with the findings of the present study.

The present study had some limitations. The results were obtained solely from studies using cell lines. The findings remain to be validated in tumor samples collected from patients with OSCC and LSCC. Expression of EPSTII in normal tissue and cancer tissue according to the patient characteristics, including the clinical stage of the cancer, tumor doubling time, and sensitivity to chemotherapy, are expected to further clarify the clinical relevance of EPSTII in these diseases. Although the comprehensive analyses with a PCR array were solely exploratory, the findings failed to explain mechanisms involved in the contrasting functions of the gene. New studies to elucidate the clinical relevance of the gene and to explore the mechanisms underlying the diverse functions of the gene according to the cancer type are warranted.

In conclusion, the present research revealed, for the first time, the opposite functions of EPSTI1, namely, of promoting and suppressing cancer progression, according to the type of cancer; i.e., its overexpression promoted the malignant phenotype in OSCC, whereas its downregulation promoted the malignant phenotype in LSCC.

\section{Acknowledgements}

Not applicable.

\section{Funding}

The present study was financially supported by grants from the Ministry of Education, Culture, Sports, Science and
Technology in Japan (Kiban-C grant nos. 17K09647 and 20K08561), and the Kanto Academic Alliance for Fostering Cancer Professionals. The abstract was presented at the 111rd Annual Meeting of the American Association for Cancer research; June 22-24 2020 in a virtual meeting, and published as abstract no. 2580 in Cancer Res 80 (Suppl 16): 2580.

\section{Availability of data and materials}

Data supporting the findings of the present study are available upon reasonable request from the corresponding author.

\section{Authors' contributions}

MF, MA, KU, MS, NK, HT and YT contributed to the design of the study. Cell culture and data acquisition were performed by MF, TC and RF. MF, MA, and AT performed statistical analyses, and interpretation of data was performed by all the authors. MF, MA and YT contributed to drafting the manuscript. All authors contributed to revising the manuscript critically for important intellectual content, as well as reading and approving the final version of the manuscript to be published.

\section{Ethics approval and consent to participate}

Not applicable.

\section{Patient consent for publication}

Not applicable. 


\section{Competing interests}

The authors declare that they have no competing interests.

\section{References:}

1. Dotto GP and Rustgi AK: Squamous cell cancers: A unified perspective on biology and genetics. Cancer Cell 29: 622-637, 2016.

2. Chi AC, Day TA and Neville BW: Oral cavity and oropharyngeal squamous cell carcinoma-an update. CA Cancer J Clin 65 401-421, 2015.

3. Torre LA, Bray F, Siegel RL, Ferlay J, Lortet-Tieulent J and Jemal A: Global cancer statistics, 2012. CA Cancer J Clin 65 : 87-108, 2015.

4. Silverman S Jr: Demographics and occurrence of oral and pharyngeal cancers. The outcomes, the trends, the challenge. J Am Dent Assoc 132 (Suppl): S7-S11, 2001.

5. Shield KD, Ferlay J, Jemal A, Sankaranarayanan R, Chaturvedi AK, Bray F and Soerjomataram I: The global incidence of lip, oral cavity, and pharyngeal cancers by subsite in 2012. CA Cancer J Clin 67: 51-64, 2017.

6. Li Y, Gu J, Xu F, Zhu Q, Ge D and Lu C: Transcriptomic and functional network features of lung squamous cell carcinoma through integrative analysis of GEO and TCGA data. Sci Rep 8: $15834,2018$.

7. Sharma B and Kanwar SS: Phosphatidylserine: A cancer cell targeting biomarker. Semin Cancer Biol 52: 17-25, 2018.

8. Loong HH, Kwan SS, Mok TS and Lau YM: Therapeutic strategies in EGFR mutant non-small cell lung cancer. Curr Treat Options Oncol 19: 58, 2018.

9. Bonner JA, Harari PM, Giralt J, Azarnia N, Shin DM, Cohen RB, Jones CU, Sur R, Raben D, Jassem J, et al: Radiotherapy plus cetuximab for squamous-cell carcinoma of the head and neck. New Engl J Med 354: 567-578, 2006.

10. Vermorken JB, Mesia R, Rivera F, Remenar E, Kawecki A Rottey S, Erfan J, Zabolotnyy D, Kienzer HR, Cupissol D, et al: Platinum-based chemotherapy plus cetuximab in head and neck cancer. N Engl J Med 359: 1116-1127, 2008.

11. Thatcher N, Hirsch FR, Luft AV, Szczesna A, Ciuleanu TE, Dediu M, Ramlau R, Galiulin RK, Bálint B, Losonczy G, et al: Necitumumab plus gemcitabine and cisplatin versus gemcitabine and cisplatin alone as first-line therapy in patients with stage IV squamous non-small-cell lung cancer (SQUIRE): An open-label, randomised, controlled phase 3 trial. Lancet Oncol 16: 763-774, 2015.

12. Nielsen HL, Ronnov-Jessen L, Villadsen R and Petersen OW: Identification of EPSTI1, a novel gene induced by epithelial-stromal interaction in human breast cancer. Genomics 79 703-710, 2002

13. Tran-Thanh D and Done SJ: The role of stromal factors in breast tumorigenicity. Am J Pathol 176: 1072-1074, 2010.

14. de Neergaard M, Kim J, Villadsen R, Fridriksdottir AJ, Rank F, Timmermans-Wielenga V, Langerød A, Børresen-Dale AL and Petersen OW: Epithelial-stromal interaction 1 (EPSTI1) substitutes for peritumoral fibroblasts in the tumor microenvironment. Am J Pathol 176: 1229-1240, 2010.

15. Kim YH, Lee JR and Hahn MJ: Regulation of inflammatory gene expression in macrophages by epithelial-stromal interaction 1 (Epsti1). Biochem Biophys Res Commun 496: 778-783, 2018.

16. Kim SC, Hong CW, Jang SG, Kim YA, Yoo BC, Shin YK, Jeong SY, Ku JL and Park JG: Establishment and characterization of paired primary and peritoneal seeding human colorectal cancer cell lines: Identification of genes that mediate metastatic potential. Transl Oncol 11: 1232-1243, 2018.

17. Hastie E, Cataldi M, Moerdyk-Schauwecker MJ, Felt SA, Steuerwald $\mathrm{N}$ and Grdzelishvili VZ: Novel biomarkers of resistance of pancreatic cancer cells to oncolytic vesicular stomatitis virus. Oncotarget 7: 61601-61618, 2016

18. Li T, Lu H, Shen C, Lahiri SK, Wason MS, Mukherjee D, Yu L and Zhao J: Identification of epithelial stromal interaction 1 as a novel effector downstream of Kruppel-like factor 8 in breast cancer invasion and metastasis. Oncogene 33: 4746-4755, 2014.

19. Capdevila-Busquets E, Badiola N, Arroyo R, Alcalde V, Soler-Lopez M and Aloy P: Breast cancer genes PSMC3IP and EPSTI1 play a role in apoptosis regulation. PLoS One 10: e0115352, 2015.

20. Fijak M and Meinhardt A: The testis in immune privilege. Immunol Rev 213: 66-81, 2006.
21. Kosova G, Scott NM, Niederberger C, Prins GS and Ober C: Genome-wide association study identifies candidate genes for male fertility traits in humans. Am J Human Genet 90: 950-961, 2012.

22. Ishii $T$, Onda $H$, Tanigawa $A$, Ohshima $S$, Fujiwara $H$, Mima $T$, Katada Y, Deguchi H, Suemura M, Miyake T, et al: Isolation and expression profiling of genes upregulated in the peripheral blood cells of systemic lupus erythematosus patients. DNA Res 12: 429-439, 2005

23. Sun JL, Zhang HZ, Liu SY, Lian CF, Chen ZL, Shao TH, Zhang S, Zhao LL, He CM, Wang M, et al: Elevated EPSTI1 promote $B$ cell hyperactivation through NF-kappaB signalling in patients with primary Sjogren's syndrome. Ann Rheum Dis 79: $518-524,2020$

24. Meng X, Yang D, Yu R and Zhu H: EPSTI1 is involved in IL-28A-mediated inhibition of HCV infection. Mediators Inflamm 2015: 716315, 2015.

25. Yamano Y, Uzawa K, Shinozuka K, Fushimi K, Ishigami T, Nomura H, Ogawara K, Shiiba M, Yokoe H and Tanzawa H: Hyaluronan-mediated motility: A target in oral squamous cell carcinoma. Int J Oncol 32: 1001-1009, 2008.

26. Peng CH, Liao CT, Peng SC, Chen YJ, Cheng AJ, Juang JL, Tsai CY, Chen TC, Chuang YJ, Tang CY, et al: A novel molecular signature identified by systems genetics approach predicts prognosis in oral squamous cell carcinoma. PLoS One 6: e23452, 2011.

27. Hou J, Aerts J, den Hamer B, van Ijcken W, den Bakker M, Riegman P, van der Leest C, van der Spek P, Foekens JA, Hoogsteden HC, et al: Gene expression-based classification of non-small cell lung carcinomas and survival prediction. PLoS One 5: e10312, 2010

28. Douglas NC and Papaioannou VE: The T-box transcription factors TBX2 and TBX3 in mammary gland development and breast cancer. J Mammary Gland Biol Neoplasia 18: 143-147, 2013.

29. Du WL, Fang Q, Chen Y, Teng JW, Xiao YS, Xie P, Jin B and Wang JQ: Effect of silencing the TBox transcription factor TBX2 in prostate cancer PC3 and LNCaP cells. Mol Med Rep 16: 6050-6058, 2017.

30. Khalil AA, Sivakumar S, Lucas FAS, McDowell T, Lang W, Tabata K, Fujimoto J, Yatabe Y, Spira A, Scheet P, et al: TBX2 subfamily suppression in lung cancer pathogenesis: A high-potential marker for early detection. Oncotarget 8: 68230-68241, 2017.

31. Ohtaki Y, Shimizu K, Kawabata-Iwakawa R, Gombodorj N, Altan B, Rokudai S, Yamane A, Kaira K, Yokobori T, Nagashima T, et al: Carbonic anhydrase 9 expression is associated with poor prognosis, tumor proliferation, and radiosensitivity of thymic carcinomas. Oncotarget 10: 1306-1319, 2019.

32. Ding M, He SJ and Yang J: MCP-1/CCL2 mediated by autocrine loop of PDGF-BB promotes invasion of lung cancer cell by recruitment of macrophages via CCL2-CCR2 axis. J Interferon Cytokine Res 39: 224-232, 2019.

33. De la Fuente Lopez M, Landskron G, Parada D, Dubois-Camacho K, Simian D, Martinez M, Romero D, Roa JC, Chahuán I, Gutiérrez R, et al: The relationship between chemokines CCL2, CCL3, and CCL4 with the tumor microenvironment and tumor-associated macrophage markers in colorectal cancer. Tumour Biol 40: 1010428318810059, 2018.

34. Kitamura T, Qian BZ, Soong D, Cassetta L, Noy R, Sugano G, Kato Y, Li J and Pollard JW: CCL2-induced chemokine cascade promotes breast cancer metastasis by enhancing retention of metastasis-associated macrophages. J Exp Med 212: 1043-1059, 2015.

35. Ling Z, Yang X, Chen X, Xia J, Cheng B and Tao X: CCL2 promotes cell migration by inducing epithelial-mesenchymal transition in oral squamous cell carcinoma. J Oral Pathol Med 48: 477-482, 2019.

36. Liu Y, Liu WB, Liu KJ, Ao L, Cao J, Zhong JL and Liu JY: Overexpression of miR-26b-5p regulates the cell cycle by targeting CCND2 in GC-2 cells under exposure to extremely low frequency electromagnetic fields. Cell Cycle 15: 357-367, 2016.

37. Lee JY, Tokumoto M, Hwang GW, Lee MY and Satoh M: Identification of ARNT-regulated BIRC3 as the target factor in cadmium renal toxicity. Sci Rep 7: 17287, 2017.

38. Watanabe J, Takiyama Y, Honjyo J, Makino Y, Fujita Y, Tateno M and Haneda M: Role of IGFBP7 in diabetic nephropathy: TGF- $\beta 1$ induces IGFBP7 via Smad2/4 in human renal proximal tubular epithelial cells. PLoS One 11: e0150897, 2016.

This work is licensed under a Creative Commons Attribution-NonCommercial-NoDerivatives 4.0 International (CC BY-NC-ND 4.0) License. 